\title{
Deformable Atlases for the Segmentation of Internal Brain Nuclei in Magnetic Resonance Imaging
}

\author{
Marius George Linguraru, Miguel Ángel González Ballester, Nicholas Ayache
}

\begin{abstract}
Magnetic resonance imaging (MRI) is commonly employed for the depiction of soft tissues, most notably the human brain. Computer-aided image analysis techniques lead to image enhancement and automatic detection of anatomical structures. However, the information contained in images does not often offer enough contrast to robustly obtain a good detection of all internal brain structures, not least the deep grey matter nuclei. We propose a method that incorporates prior anatomical knowledge in the shape of digital atlases that deform to fit the image data to be analysed. Our technique is based on a combination of rigid, affine and non-rigid registration, segmentation of key anatomical landmarks and propagation of the information of the atlas to detect deep grey matter nuclei. The Montreal Neurological Institute (MNI) and Zubal atlases are employed. Results show that detecting important structures such as the ventricles and brain outlines greatly improves the results. Our method is fully automatic.

Keywords: MRI, brain, deep grey matter nuclei, atlas, image normalisation, registration, segmentation.
\end{abstract}

\section{Introduction}

The advent of medical imaging modalities such as X-ray, ultrasound, computed tomography (CT) and magnetic resonance imaging (MRI) has greatly improved the diagnosis of various human diseases. To date, the most common procedure to analyse imaging data is visual inspection on printed support. In the last decade, computer-aided medical image analysis techniques have been employed to provide a better insight into the acquired image data. [5]. Such techniques allow for quantitative, reproducible observation of the patient condition. Furthermore, the computing power of modern machines can be used to combine information from several images of the same patient (i.e. image fusion) or add prior information from a database of images.

In this paper, we present a fully automated medical image analysis technique aimed at the detection of internal brain structures from MRI data. Such automated processes allow the study of large image databases and provide consistent measurements over the data. In our case, we employ a priori anatomical knowledge in the form of digital brain atlases.

Relevant background information about MRI and brain anatomy is provided next. Section 2 will describe the different components of our image processing framework, which detects and delineates internal brain structures by identifying analogous structures in digital brain atlases. Finally, results and conclusions are given.

\subsection{Magnetic Resonance Imaging}

MRI has become a leading technique widely used for imaging soft human tissue. Its applications are extended over all parts of the human body and it represents the most common visualisation method of human brain. Images are generated by measuring the behaviour of soft tissue under a magnetic field. Under such conditions, water protons enter a higher energy state when a radio-frequency pulse is applied and this energy is re-emitted when the pulse stops (a property known as resonance) [7]. A coil is used to measure this energy, which is proportional to the quantity of water protons and local biochemical conditions. Thus, different tissues give different intensities in the final MR image. From the brain 
MRI perspective, this quality makes possible the segmentation of the three main tissue classes within the human skull: grey matter (GM), white matter (WM) and cerebrospinal fluid (CSF). Their accurate segmentation remains a challenging task in the clinical environment.

The relative contrast between brain tissues is not a constant in MR imaging. In most medical imaging applications, little can be done about the appearance of anatomically distinct areas relative to their surroundings. In MRI, the choice of the strength and timing of the radio-frequency pulses, known as the MRI sequence [12], can be employed to highlight some type of tissue or image out another, according to the clinical application. However, the presence of artefacts due to magnetic field inhomogeneity (bias fields) and movement artefacts may hamper the delineation of GM versus WM and CSF and make their depiction difficult.

There is an entire family of MRI sequences that are used in common clinical practice. T1-weighted MRI offers the highest contrast between the brain soft tissues. On the contrary, T2-weighted and Proton Density (PD) images exhibit very low contrast between GM and WM, but high contrast between CSF and brain parenchyma. In other MRI sequences, like the Fluid Attenuated Inversion Recovery (FLAIR) sequence, the CSF is eliminated from the image in an adapted T1 or T2 sequence. More about these specific MRI sequences and their variations can be found in [2]. Multisequence MRI analysis combines the different information provided by the employed sequences. Combining such knowledge gives substantially more information about brain anatomy and possible occurring changes.

MR images depict a 3D volume where the organ or part of the body of interest is embedded. This information can be used to build a 3D representation of the structure of interest. This applies both to 2D sequences, where images are acquired in slices, and to recently developed 3D sequences, where the data are captured in the 3D Fourier space, rather than each slice being captured separately in the 2D Fourier space $[2,12]$.

\subsection{Deep Grey Matter Nuclei}

The neurones that build up the human brain are composed of a cellular body and an axon. The latter projects its dendritic connections to other neurones in remote cerebral regions. In essence, grey matter corresponds to the cellular bodies, whereas the axons constitute the white matter. Cerebral grey matter is mainly concentrated in the outer surface of the brain (cortex), but several internal GM structures exist, as seen in Figures 1 and 2. These are known as deep grey matter nuclei and they play a central role in the intellectual capabilities of the human brain. Additionally, deep brain grey matter nuclei are relevant to a set of clinical conditions, such as Parkinson's and Creutzfeldt-Jakob diseases. However, their detection in MRI data sets remains a challenging task, due to their small size, partial volume effects [6], anatomical variability, lack of white matter-grey matter contrast in some sequences and movement artefacts. A methodology for the robust detection of deep brain grey matter nuclei in multi-sequence MRI is presented in this paper.

\section{Method}

\subsection{Spatial Normalisation}

The large variability inherent to human anatomy and the differences in patient positioning across scans leads us to consider spatial normalisation as an approach to put patient images in a standard reference frame. This will allow to localise the areas of interest with the help of an atlas of the brain. Furthermore, it will make automatic inter-patient comparisons possible.

The identification of brain structures in volumetric images can be automated thanks to the use of digital atlases. These are images that have been segmented and thus contain information about the position and shape of each structure. Such atlases can be binary ( 1 for the location of a structure and 0 


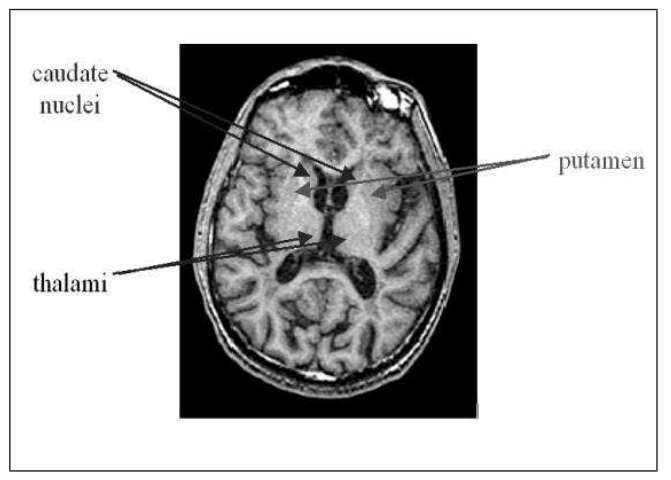

Figure 1: Deep grey matter internal nuclei as seen in a normal T1 weighted axial MR image with good contrast between WM, GM and CSF. The arrows point towards some of these nuclei, namely the caudate nuclei, the thalami and the putamen.

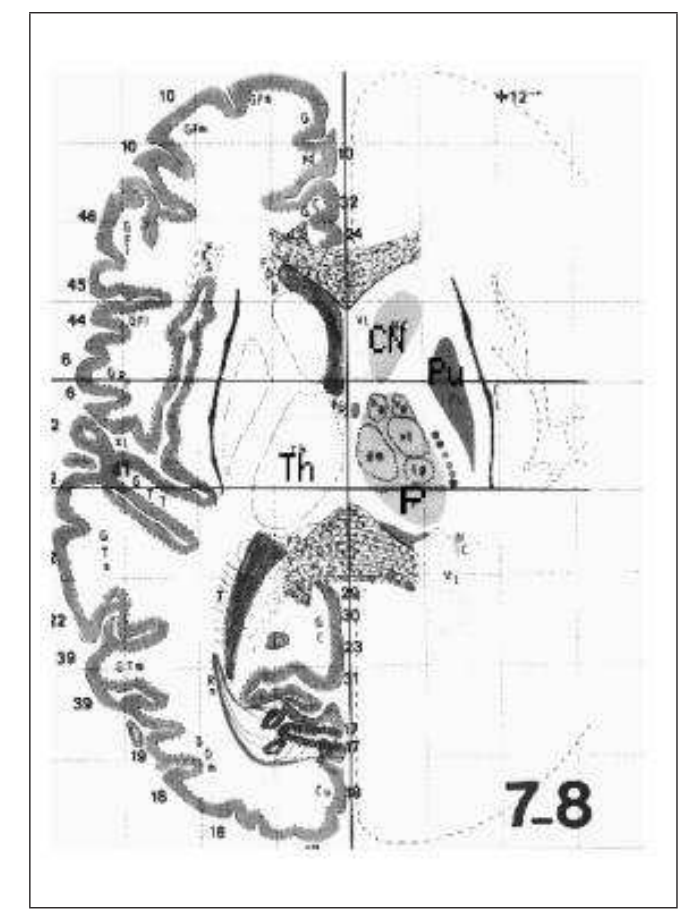

Figure 2: An annotated map of deep grey matter internal nuclei reproduced from the Talairach and Tournoux atlas [13]: the caudate nuclei $(\mathrm{CN})$, putamen $(\mathrm{Pu})$ and thalami $(\mathrm{Th})$. 
for "outside") or probabilistic, in which case the values correspond to the probability of a voxel containing the structure of interest. In order to locate such structures in a given patient image, the atlas image is deformed to match the shape of the patient brain. This process is known as registration. Depending on the type of geometric deformation allowed, registration can be rigid, affine, parametric (e.g. spline) or free-form (a deformation field specifying the displacement applied to each point).

Registration to a digital atlas has become a common technique with the introduction of popular statistical algorithms for image processing, such as Statistical Parametric Mapping (SPM) [1] or Expectation Maximization Segmentation (EMS) [14]. A well-known probabilistic atlas in the scientific community is the MNI Atlas from the Montreal Neurological Institute at McGill University [4]. It was built using over 300 MRI scans of healthy individuals to compute an average brain MR image, the MNI template, which is now the standard template of SPM and the International Consortium for Brain Mapping [9]. The averaging is performed for the entire brain, but also on isolated GM, WM and CSF, providing a tool for statistical segmentation. For these reasons, we chose the MNI template as the basis for image alignment in our approach. Figure 3 shows the MNI template.

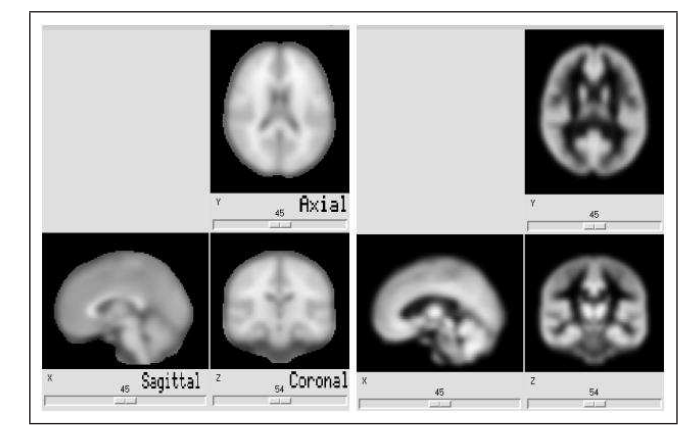

Figure 3: The MNI template. On the left, the probabilistic MNI atlas of the brain; on the right, the corresponding GM atlas. Please note the arrangement of MR images in radiological convention with an axial, a sagittal and a coronal view. This convention is reflected in figures throughout the paper.

We propose the following registration scheme. T1 images have often the highest resolution, hence we register them to the MNI template first using an affine transformation. The registration algorithm, previously developed in our group, is described in [11]. It uses a block matching strategy in a twostep iterative method. The standard assumption behind the algorithm is that there is a global intensity relationship between the template image and the one being registered to it. The method proposes several types of correlation measures: linear, functional or statistical. Maximising one of these, the correlation coefficient in our case, the transformation between the two images is computed block by block and a displacement field is thus generated. A parametric transformation, either affine or rigid, is then estimated from this deformation field. To further improve robustness, this procedure is repeated at multiple scales. More details can be found in [10].

Next, rigid intra-patient registration of all sequences is performed using the same algorithm as above. T2, FLAIR, diffusion-weighted, diffusion tensor or other sequence images can be registered to the T1 image. Since this registration is performed on images of the same patient acquired during the same scanning session, rigid registration suffices. By combining these rigid transformations with the affine transformation matching T1 and MNI template, we can find correspondences between the atlas and the other sequence images. This is illustrated in Figure 5. The final image resolution is that of the MNI atlas: 9110991 voxels. Figure 4 shows an example of spatial normalisation. With all images registered to the atlas, intra- and inter-patient analysis becomes simple and statistical algorithms can be applied. 


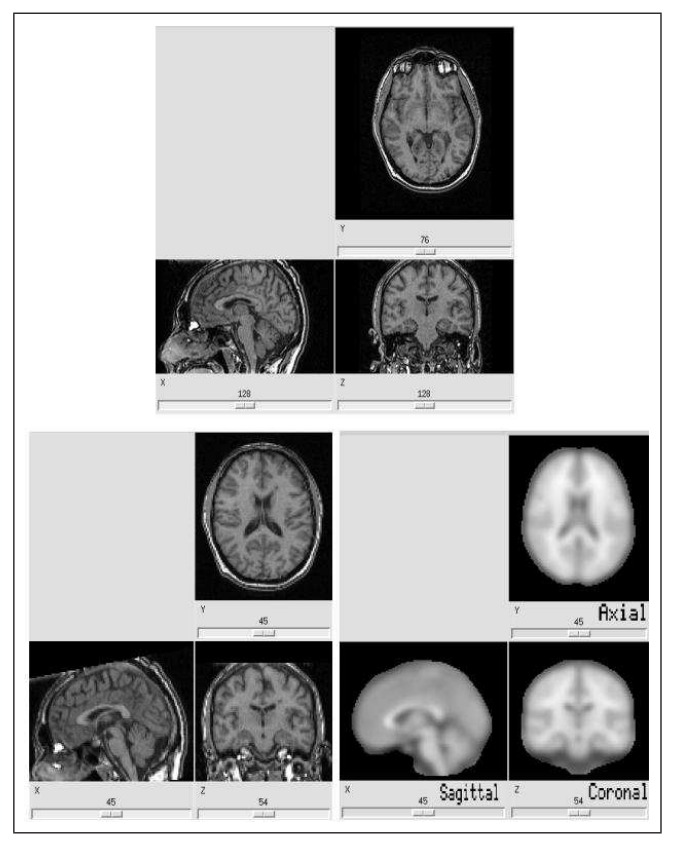

Figure 4: An example of spatial normalisation. The image on top is the subject's T1 before registration; the image on bottom left shows the subject's T1 after spatial normalisation and the MNI template is presented on the bottom right image.

\subsection{A Priori Anatomical Knowledge}

To be able to segment GM and WM in MRI sequences, a good contrast between these types of tissue in T1-weighted images is desired. Figure 6 shows a typical T1 with high contrast between brain soft tissues and a common T1 image from our database. Under the given circumstances, the segmentation of GM cannot be done directly from the patient images. The MNI atlas can provide a probabilistic segmentation of GM, but this is not precise enough for our application. We use instead a segmented anatomical atlas of the brain, the Zubal Phantom [15], which is introduced next.

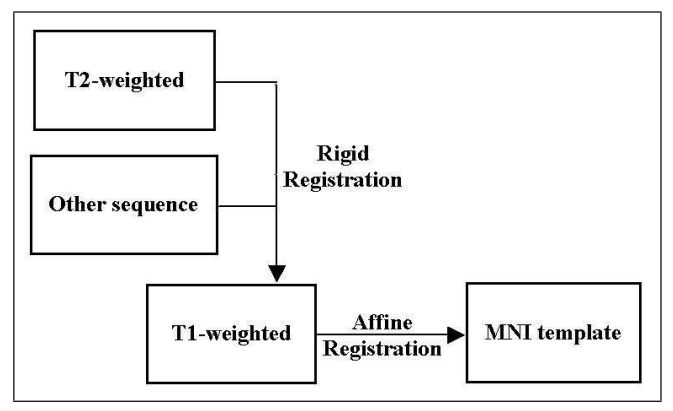

Figure 5: Diagram of the spatial normalisation algorithm. Intra-patient images are rigidly registered on the corresponding T1. The T1-weighted image is affinely registered to the atlas template. The resulting transformation is used to align all other MR images to the atlas.

The Zubal atlas offers a precisely labelled segmentation of brain structures from the T1-weighted MR image of one single subject. Our interest focuses on the internal nuclei, which are segmented in the phantom. First, the atlas must be aligned to our set of images, which have been previously registered to the MNI atlas. Thus, we register the Zubal Phantom to the MNI template, again using our block matching algorithm [11], to estimate an affine transformation. However, in order to preserve the correct 


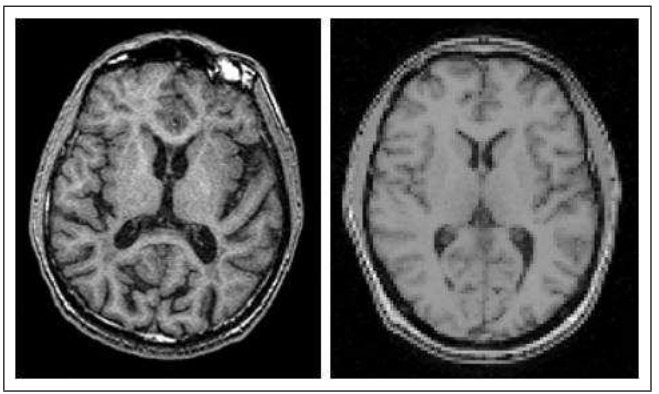

Figure 6: A typical T1-weighted MR image with good contrast between brain GM, WM and CSF (left) versus a T1 image where GM and WM cannot be reliably distinguished from each other.

values of the segmentation labels posterior to the application of the transformation, nearest-neighbour interpolation is performed, as opposed to the case of patient image registration, which employed spline interpolation. Figure 7 shows the results of registering the Zubal Phantom to the MNI reference without disrupting the Zubal labels.

\subsection{Refined Segmentation}

Once the Zubal Phantom is registered to the working framework, we can easily depict the brain structures that are of interest, namely the deep GM internal nuclei. For the examples in this paper, we will focus on the basal ganglia. Hence, we create a mask with the thalamus, putamen and head of the caudate - which will be referred as internal nuclei for the rest of this paper - from the Zubal Phantom registered on MNI (Figure 8). We aim to use this mask for the segmentation of internal nuclei in patient images. Although the affine registration gives correct correspondences in a general brain registration framework, the anatomical variability between patients makes the correspondence between the Zubal internal nuclei mask and the corresponding internal nuclei in each patient erroneous. A refinement of the registration in the deep GM between the Zubal internal nuclei mask and the patient internal nuclei seems necessary to allow us to use the a priori anatomical information resulting from the segmentation of the Zubal Phantom.

The segmentation of internal nuclei in patient images is not an obvious task; this is why we exploit the Zubal Phantom. Nevertheless, there are other important anatomical landmarks in the brain that are easier to identify. We concentrate on the segmentation of ventricles and cortex external boundary. Ventricles will give a good approximation of the deformation field around the internal nuclei, whereas the cortex boundary will impose the global spatial correspondence and stabilise the deformation field inside the brain. Figure 8 illustrates the segmentation of ventricles, brain contour and internal nuclei from the registered Zubal Phantom.

To obtain similar images of segmented brain margin and ventricles for each patient, we employ morphological opening on patient T2 images. The strong contrast that CSF has against the brain in T2weighted images allows us to segment the ventricles, while the cortex boundary can be extracted from either $\mathrm{T} 1$ or $\mathrm{T} 2$ sequences. We prefer using the $\mathrm{T} 1$ sequence, since the T2 image we employ lacks some top and bottom slices. The ventricles being located in the middle of the brain, it is correct to extract them from T2 images, but the cortex would be incomplete.

We are now in the possession of two binary maps of ventricles and brain boundaries for each patient: one from the Zubal Phantom and the other from the patient. Non-rigid (free-form) registration is used to align the two images, employing the algorithm developed in our group and described in [3]. This registration method minimises an energy function, which uses measures of intensity similarity, smoothing, noise parameters and correspondence between points. Figure 9 shows typical results and Figure 10 shows the 3D deformation fields related to the registration in Figure 9. The outer margin of the cortex ensures that 


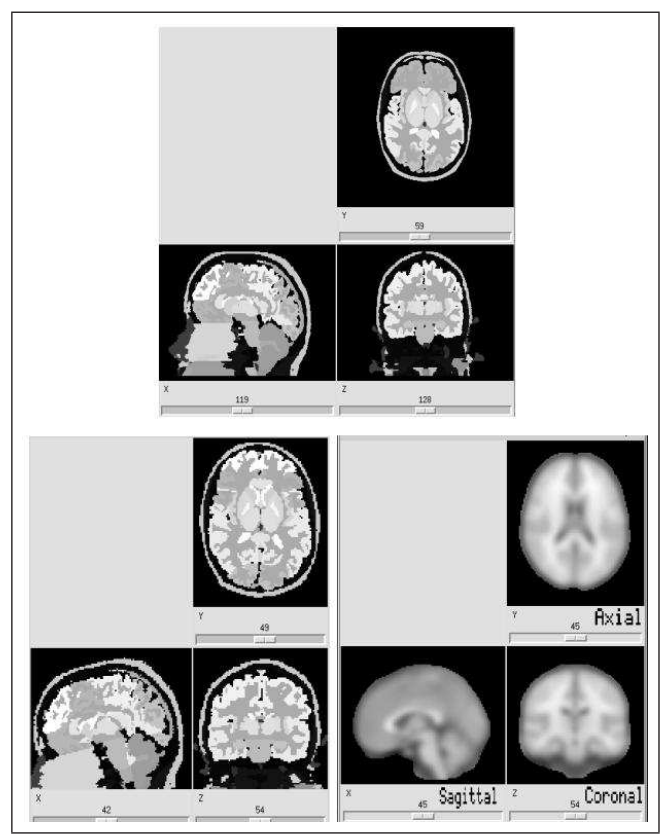

Figure 7: The registration of Zubal Phantom onto the MNI template. On the top row, the original Zubal Phantom is shown; on the bottom- left, we have the registered Zubal Phantom on the MNI template, which is shown in the bottom- right image.

the deformation fields are spatially sound and do not pull the internal nuclei over their location.

Having the deformation fields computed, we apply them to the mask of internal nuclei of the Zubal Phantom, deforming the mask according to the position and size of the ventricles in the patient image. A diagram of the algorithm is shown in Figure 11. The deformed mask is used to segment the internal nuclei of the patient, namely the putamen, head of the caudate and thalamus.

Figure 12 shows an example of registration of internal nuclei in 3D and the internal nuclei segmentation results in a T1-weighted MR image of a patient. In Figure 13 we segment the internal nuclei in a patient T2-weighted image. The segmentation can be accurately performed in any MR sequence of the patient, given that multisequence images have been previously registered to the MNI atlas.

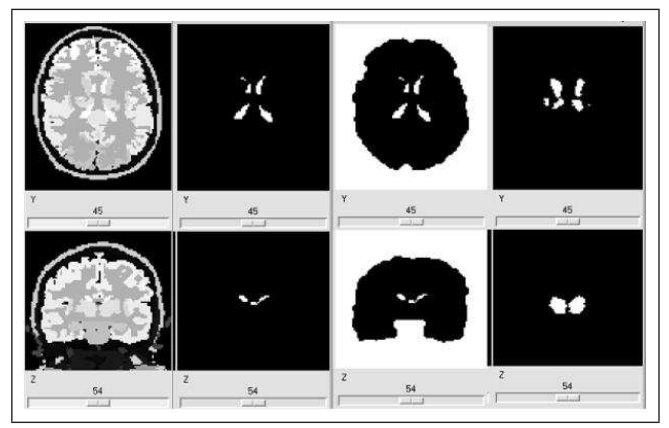

Figure 8: The segmentation of the Zubal Phantom. From left to right: column 1, the Zubal Phantom registered on MNI; column 2, the ventricles segmented from the Zubal Phantom; column 3, the cortex outer boundary is added to the ventricles; column 4, the internal nuclei segmented from the Zubal Phantom. The top row shows the axial view, while on bottom we present the coronal view.

In this paper, we focused on the segmentation of the basal ganglia to present our algorithm for the segmentation of deep grey matter nuclei. An identical approach can be used for other inner brain 
structures to accurately segment them in patient images. Each nuclei class has an associated label in the Zubal Phantom, which facilitates their identification. In Figure 14 we illustrate the segmentation of individual types of nuclei, here caudate nuclei, thalami and putamen, using our approach.

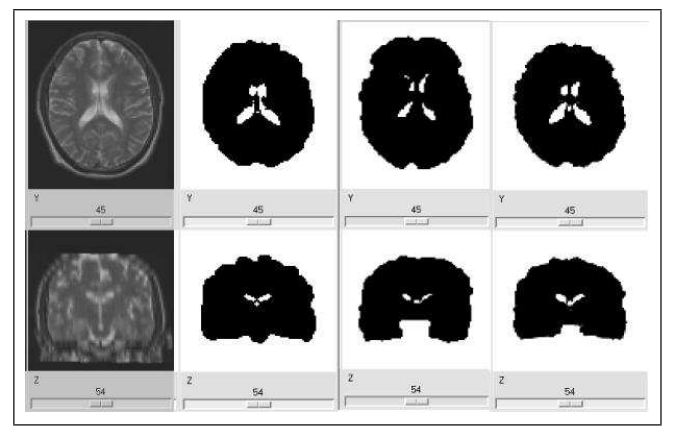

Figure 9: Registration of the Zubal ventricles and cortex outer boundary on a patient. The patient's ventricles are larger next to the small ventricles in the Zubal Phantom, where the subject is young. The algorithm gives robust results, as seen above. From left to right: column 1, the T2-weighted image of the patient; column 2, the ventricles and brain margin of the patient (ventricles segmented from T2 and cortex from T1); column3, the ventricles and brain boundary of Zubal Phantom; column 4, the ventricles and cortex boundary of the Zubal Phantom registered on the patient.

\section{Conclusion}

A robust automatic technique for the identification of deep brain internal nuclei was presented. The use of key anatomical landmarks such as the ventricles and the outline of the brain imposes anatomical constraints in the deformation fields found by the non-rigid registration algorithm, which otherwise would fail to converge to the correct segmentation.

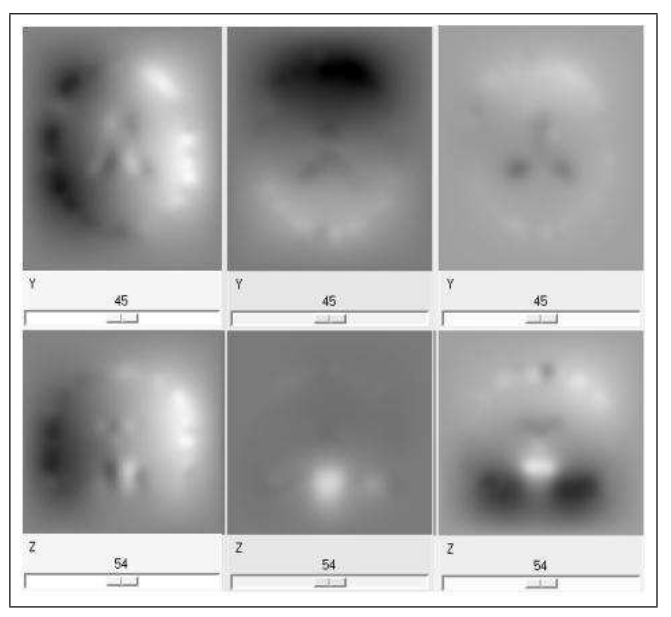

Figure 10: Deformation fields of the non-rigid registration between the Zubal Phantom ventricles and those of a patient with very large ventricles. On the left is the $\mathrm{x}$ field, the $\mathrm{y}$ field is in the middle column and the $\mathrm{z}$ field on the right. 


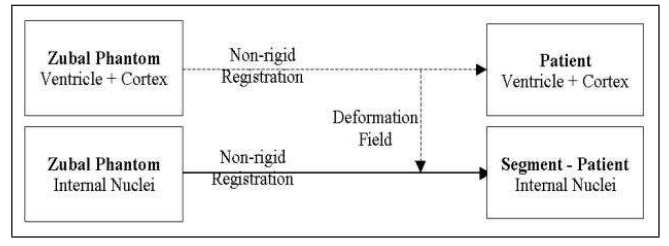

Figure 11: Diagram of the refined registration of internal nuclei.

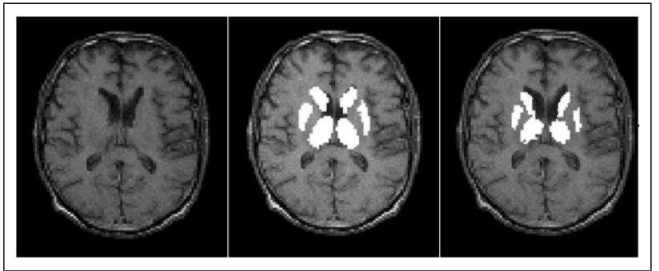

Figure 12: An example of internal nuclei registration and their segmentation in a T1-weighted patient image. On the left, we have the T1 image of the patient; in the middle column, we show the segmentation of internal nuclei according to the binary map before non-rigid deformation with the head of the caudate superposed on the ventricle; on the right, we segment the internal nuclei after non-rigid deformation, showing an accurate segmentation.

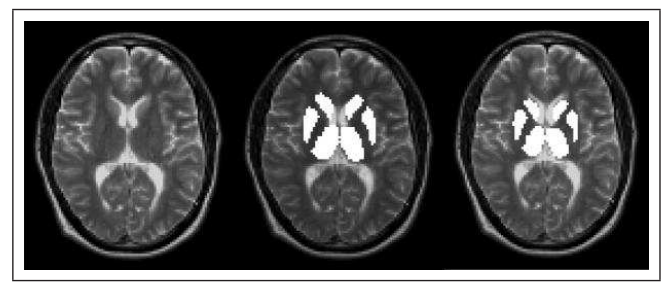

Figure 13: An example of internal nuclei segmentation in a T2-weighted image of the patient. On the left, we have the T2 image of the patient; in the middle column, we show the segmentation of internal nuclei before non-rigid deformation; on the right, we segment correctly the internal nuclei after non-rigid deformation.

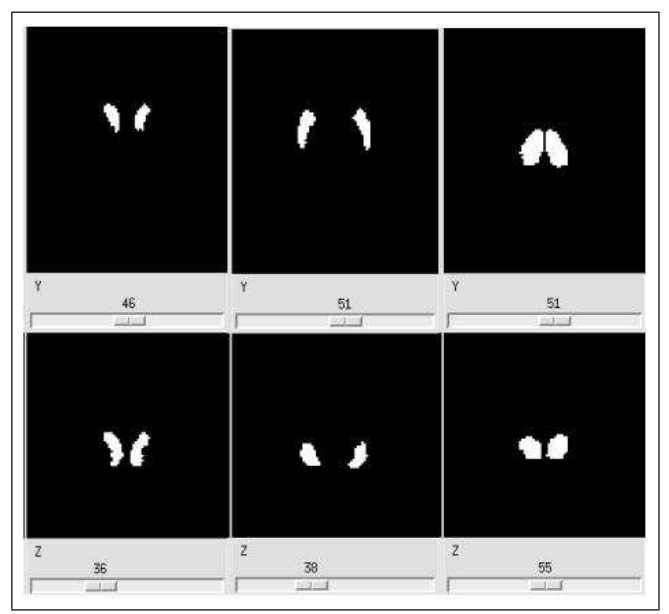

Figure 14: Binary maps of deep grey nuclei. From left to right: the caudate nuclei, the putamen and the thalami. From top to bottom: axial and coronal views. These individual masks can be used for the accurate segmentation of each type of nuclei. 


\section{Acknowledgement}

The authors would like to thank Professor Ioana Moisil from the "Lucian Blaga" University of Sibiu for her assistance.

\section{References}

[1] J. Ashburner, K.J. Friston, "Voxel-Based Morphometry - The Methods", NeuroImage, 11:805-821, 2000.

[2] M A. Brown, R.C. Semelka, "MR Imaging Abbreviations, Definitions, and Descriptions: A Review", Radiology, 213:647-662, 1999.

[3] P. Cachier, E. Bardinet, D. Dormont, X. Pennec, N. Ayache, "Iconic Feature-based Nonrigid Registration: The PASHA Algorithm", CVIU - Special Issue on Nonrigid Registration, 89(2-3):272-298, 2003.

[4] D.L. Collins, A.P. Zijdenbos, V. Kollokian, J.G. Sled, N.J. Kabani, C.J. Holmes, A.C. Evans, "Design and Construction of a Realistic Digital Brain Phantom", IEEE Transactions on Medical Imaging, 17(3):463-468, 1998.

[5] J. Duncan, N. Ayache, "Medical Image Analysis: Progress over Two Decades and the Challenges Ahead", IEEE Transactions on Pattern Analysis and Machine Intelligence, 22(1):85-106, 2000.

[6] M. A. González Ballester, A. Zisserman, M. Brady, "Estimation of the Partial Volume Effect in MRI", Medical Image Analysis, 6(4):389-405, 2002.

[7] J P. Hornak, "The Basics of MRI", http://www.cis.rit.edu/htbooks/mri/.

[8] M.G. Linguraru, M.A. Gonzales Ballester, E. Bardinet, D. Galanaud, D. Dormont, J.P. Brandel, N. Ayache, "Automated Analysis of Basal Ganglia Intensity Distribution in Multisequence MRI of the Brain - Application to Creutzfeldt-Jakob Disease", Rapport de Recherche, INRIA, 2004.

[9] J.C. Mazziotta, A.W. Toga, A.C. Evans, P.T. Fox, J. Lancaster, K. Zilles, R.P. Woods, T. Paus, G. Simpson, B. Pike, C.J. Holmes, D.L. Collins, P.M. Thompson, D. MacDonald, M. Iacoboni, T. Schormann, K. Amunts, N. Palomero-Gallagher, S. Geyer, L. Parsors, K.L. Narr, N. Kabani, G. Le Goualher, M. Boomsma, T. Cannon, R. Kawashima, B. Mazoyer, "A Probabilistic Atlas and Reference System for the Human Brain: International Consortium for Brain Mapping (ICBM)", Philosophical Transactions of the Royal Society of London, Series B (Biological Sciences), 356(1412):1293-1322, Appendix II, 2001.

[10] S. Ourselin, "Recalage d'Images Médicales par Appariement de Régions - Application à la Construction d'Atlas Histologiques 3D" , PhD thesis, Université de Nice - Sophia Antipolis, 2002.

[11] S. Ourselin, A. Roche, S. Prima, N. Ayache, "Block Matching: A General Framework to Improve Robustness of Rigid Registration of Medical Images", in A.M. DiGioia and S. Delp (eds.) Medical Robotics, Imaging and Computer Assisted Surgery (MICCAI 2000), volume 1935 of Lectures Notes in Computer Science, Springer, 557-566, 2000.

[12] D.D. Stark, W.G. Bradley, W.G. Jr. Bradley, "Magnetic Resonance Imaging", Mosby, 1999.

[13] J. Talairach, P. Tournoux, "Co-Planar Stereotaxic Atlas of the Human Brain", Thieme Medical Publishers, 1988. 
[14] K. Van Leemput, F. Maes, D. Vandermeulen, A. Colchester, P. Suetens, "Automated Segmentation of Multiple Sclerosis Lesions by Model Outlier Detection", IEEE Transactions on Medical Imaging, 20(8):677-688, 2001.

[15] I.G. Zubal, C.R. Harrell, E.O. Smith, Z. Rattner, G. Gindi, P.B. Hoffer, "Computerized Threedimensional Segmented Human Anatomy", Medical Physics, 21:299-302, 1994.

Marius George Linguraru EPIDAURE/ASCLEPIOS Research Group, INRIA

Sophia Antipolis, France

Division of Engineering and Applied Sciences

Harvard University, Cambridge MA, USA

E-mail: mglin@deas.harvard.edu

Miguel Ángel González Ballester University of Bern, Bern, Switzerland

MEM Research Center, Institute for Surgical Technology and Biomechanics

Nicholas Ayache

EPIDAURE/ASCLEPIOS Research Group, INRIA

Sophia Antipolis, France

Received: November 16, 2006

Editor's note about the author:

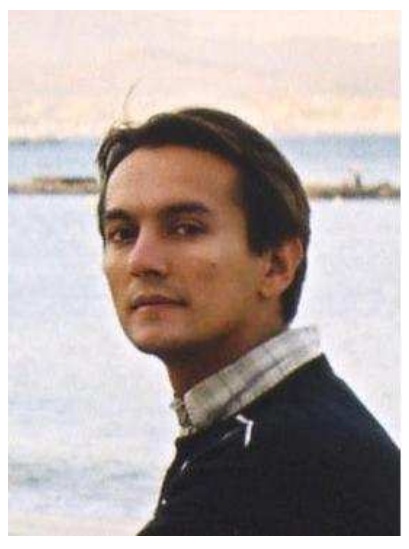

Marius George LINGURARU joined the Diagnostic Radiology Department at the Clinical Center at the National Institute of Health (NIH), Bethesda, Maryland, USA in 2007 as Staff Scientist. Previously, he worked as Research Fellow in the Division of Engineering and Applied Sciences of Harvard University in Cambridge, Massachusetts, USA. He moved to the Boston area from the South of France, where he was Expert Engineer in the Epidaure/Asclepios Research Group of the National Institute of Research in Informatics and Automatic Control (INRIA) in Sophia Antipolis, France. He received a $\mathrm{PhD}$ in Information Engineering/Medical Image Analysis at the University of Oxford, Oxford, UK within the Medical Vision Laboratory and was a member of Keble College. His previous studies include an MA in British Cultural, an MSc in Parallel and Distributed Processing Systems, Studies and a BSc in Computer Science. All three degrees are from the "Lucian Blaga" University of Sibiu, Romania, where he also worked as Assistant Professor in the Department of Computer Science and Automatic Control. 